\title{
Clinical Observation and Proposed Classification of Vitiliginous Patches by a Wood's Lamp
}

\author{
Yuka Kimura*, Atsushi Tanemura*\# (D), Yukiho Kurosaki, Madoka Takafuji, Kazunori Yokoi, \\ Eiji Kiyohara, Noriko Arase, Manabu Fujimoto
}

Department of Dermatology, Osaka University Graduate School of Medicine, Suita, Japan

Email: \#tanemura@derma.med.osaka-u.ac.jp

How to cite this paper: Kimura, Y., Tanemura, A., Kurosaki, Y., Takafuji, M., Yokoi, K., Kiyohara, E., Arase, N. and Fujimoto, M. (2020) Clinical Observation and Proposed Classification of Vitiliginous Patches by a Wood's Lamp. Journal of Cosmetics, Dermatological Sciences and Applications, 10, 204-211.

https://doi.org/10.4236/jcdsa.2020.104021

Received: November 15, 2020

Accepted: December 14, 2020

Published: December 17, 2020

Copyright $\odot 2020$ by author(s) and Scientific Research Publishing Inc. This work is licensed under the Creative Commons Attribution International License (CC BY 4.0).

http://creativecommons.org/licenses/by/4.0/

\begin{abstract}
Although vitiligo lesion especially in static state is characterized as sharply demarcated and complete depigmented macule, we encounter patients who have various manners of hypopigmented lesions. We examined the 81 lesions using the newly released Wood's lamp (Woody ${ }^{\otimes}$ ) and investigated whether or not vitiliginous lesions could be uniformly classified under Wood's lamp illumination and also this classification helped to estimate the tendency of repigmentation after treatment. As result, the vitiliginous lesions were categorized into 4 types on intra- and peri-lesions prior to treatment by using the Wood's lamp. The inside and border of the lesions were classified as follows: clear white, faint, multi-dot, and perifollicular for the inside, and sharp, blunt, confetti, and trichrome for the border. Suggestive residual pigmentation was detected in $73.6 \%$ of patients at the first visit and repigmentation was observed in $67.9 \%$ of patients at least 3 months after treatment. Lesions with the "clear white" inside pattern showed significantly lower repigmentation frequency in $38.5 \%$ of patients compared to others. The borders with 4 enlarged lesions were composed of 3 of confetti-type and one of sharp-type. This preliminary study demonstrated that detailed observation with a Wood's lamp could be the basis to classify vitiliginous lesions and might be useful for predicting not only disease progression but also repigmentation prior to treatment.
\end{abstract}

\section{Keywords}

Vitiligo, Wood's Lamp, Classification, Repigmentation, Clinical Course

\section{Introduction}

Vitiligo is an acquired hypopigmentary disorder characterized by the progressive

${ }^{\star}$ These authors equally contributed to this work. 
loss of melanocytes and the lesions represent complete or near depigmenation with a well-defined border in its extensive form, vitiligo universalis [1]. However, dermatologists often experience heterogeneity among lesions as fuzzy inside and indistinct border.

Since ultraviolet (UV) light photography is based on the principle that UV rays are more selectively absorbed by melanin than visible light [2] [3] [4], the differences between depigmented lesions can be distinguished more clearly via observation under a Wood's lamp than under a normal lamp. It is more important than ever to define a progressive versus stable status and predict the treatment efficacy for each vitiliginous patch, not only visually but also with the help of a Wood's lamp, with recent developments in therapeutic options for vitiligo. However, no investigations have systematically and sequentially observed vitiligo lesions in detail with follow-up of the clinical course.

Observation under UV-A light at a narrow wavelength peak of 365 to $385 \mathrm{~nm}$ with a Wood's lamp enables the differentiation of healthy sites and depigmented spots with stronger contrast than conventional UV light using a fluorescent lamp [5], with the development of light-emitting diode optical technology. We previously reported that observation under a Wood's lamp was useful for discriminating vitiligo from nevus depigmentosus in pediatric patients, based on clinical course and the lesional feature under UV light [6].

In this investigation, we attempted to classify the patterns of vitiligo lesions by using the newly released Wood's lamp (Woody ${ }^{\circledR}$; USHIO Inc., Tokyo, Japan) that emits UV light at a 365 - to $385-\mathrm{nm}$ wavelength, as well as based on visual findings. It is expected that the Woody potentiates to detect depigmentation spots more clearly than conventional UV light because of equivalently covering the whole view field and the attached $\mathrm{x} 2$ magnifying clear glass. Furthermore, we retrospectively evaluated the correlation of each classified pattern with the clinical course, treatment response, and repigmentation type.

\section{Methods}

A total of 81 vitiligo lesions selected one by one from the 81 cases were assessed for this study. Table 1 shows the demographics of the patients enrolled in this study. We retrospectively analyzed the following items: gender, age, clinical type, observation site, and visual features under Wood's lamp illumination before, during, and after treatment.

Vitiliginous patches were classified into 4 types, regarding the type of interior and border of each lesion observed under a Wood's lamp, as follows: clear white, faint, multi-dot, and perifollicular. No supposed residual pigmentation was observed in the "clear white" type even under Wood's light. However, while the "faint" type included lesions with even incomplete and fuzzy hypopigmentation, the "multi-dot" type included lesions predominantly with irregular pigmented islands, and the "perifollicular" type included perifollicular pigmentation (Figure 1(a)). The border was also classified into 4 patterns: sharp, blunt, confetti, and 
trichrome. "Sharp" referred to lesions with a sharp boundary, while "blunt" lesions were slightly blurred and indistinct. "Confetti" referred to confetti-like lesions, and "trichrome" had an intermediate color between vitiligo and the healthy sites, with the border appearing to be of 3 colors (Figure 1(b)). The repigmentation type was classified into perifollicular, marginal, diffuse, or mixed, following treatment. "Perifollicular" referred to lesions with follicular pigmentation spots, "marginal" referred to lesions that had shrunk from the border, "diffuse" referred to lesions with overall incomplete repigmentation, and "mixed"
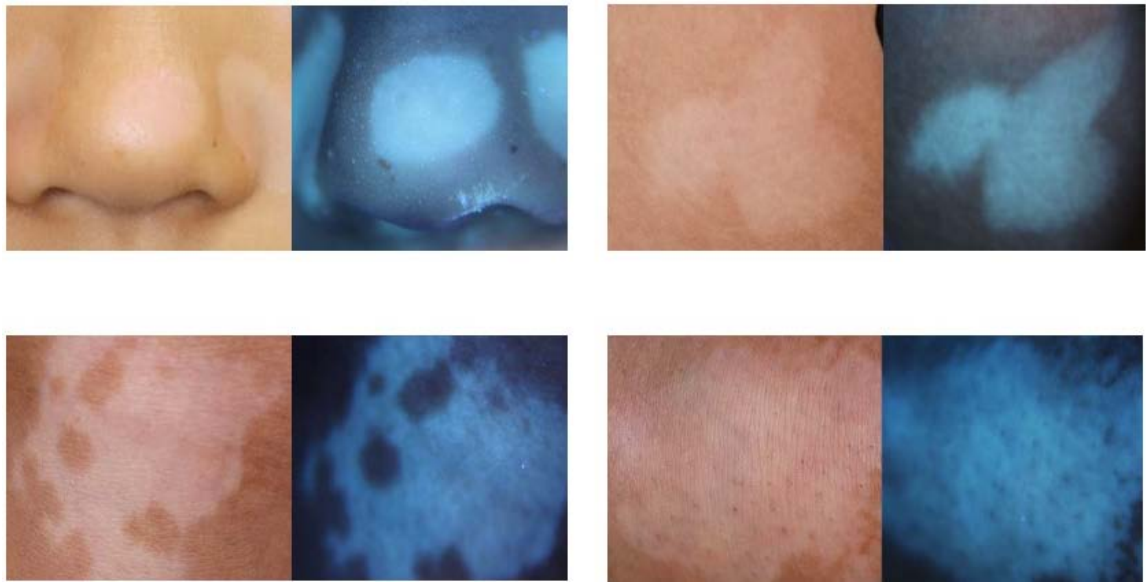

(a)
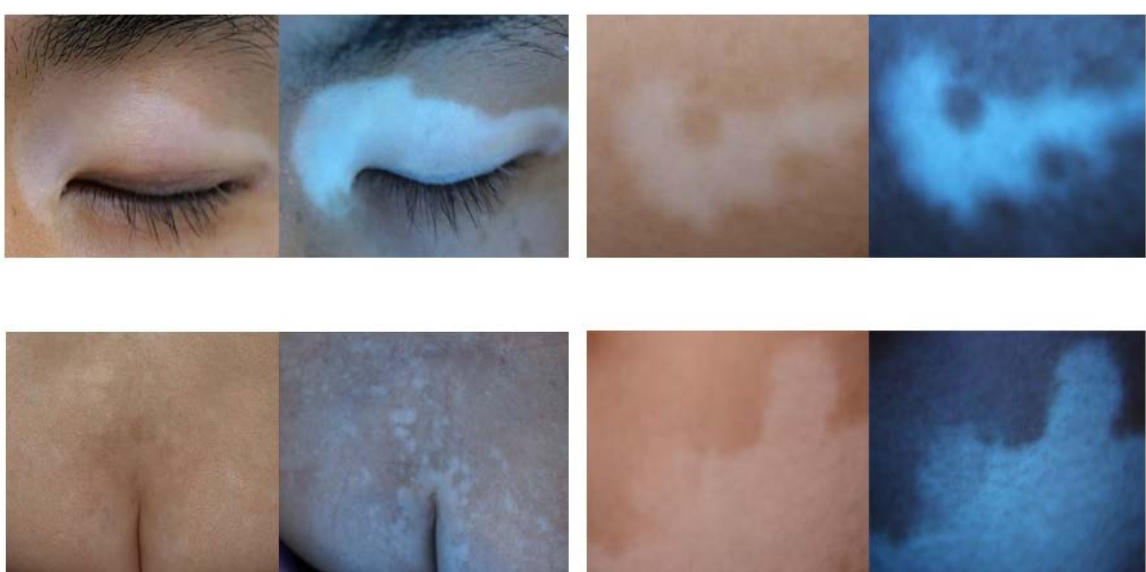

(b)

Figure 1. (a) Initial inner lesions were classified into four types. Complete hypopigmented lesion was classified to "clear white" type (left upper panels). Even incomplete and fuzzy hypopigmented lesion was classified to "faint" type (right upper panels). Lesion with multi-sized pigmented islands was classified to "multi-dots" type (left lower panels). Lesions with consistent perifollicular pigmentation were classified to "perifollicular" type (right lower panel). Left and right images are visual and under Wood's lamp view, respectively. (b) Initial lesions' border was also classified into four patterns. Lesions with sharp boundary were classified to "sharp" pattern (left upper panels). "blunt" pattern was with slightly blurred and indistinct border (right upper panels). "confetti” pattern referred to confetti-like lesions (left lower panels) and "trichrome" pattern had an intermediate color between vitiligo and healthy sites, with the border appearing to be of three colors (right lower panels). Left and right images are visual and under Wood's lamp view, respectively. 
Table 1. (a) Enrolled patients' profile and suggestive pattern of vitiligo lesions under Wood's lamp observation; (b) Relationship between the initial inside feature and repigmentation pattern under Wood's lamp observation.

(a)

n (\%)

Gender

Female

$42(51.9)$

Male

$39(48.1)$

Age

ranged from

1 to 78 yrs (mean age 28.4)

Clinical type

Segmental

$36(44.4)$

Nonsegmental

$30(37.1)$

Unclassified

15 (18.5)

Lesion sites for observation

Face/neck

Trunk

$22(27.2)$

Limb

Acral

Treatment option

Topical

Steroid alone

TCI (topical calcineurin inhibitor) alone

$1(1.5)$

Vit. D3 alone

5 (7.6)

Combined

23 (34.8)

with phototherapy*

$22(33.3)$

Systemic steroid

Initial feature

Inside

Clear white

$13(16.9)$

Faint

22 (28.6)

Multi-dot

$26(33.8)$

Perifollicular

$16(20.8)$

Border

Sharp

23 (29.9)

Blunt

40 (51.9)

Confetti

$11(14.3)$

Trichrome

53 out of 72

Frequency of each repigmentation pattern

$10(18.9)$

Marginal

7 (13.2)

Diffuse

$5(9.4)$

Perifollicular

$31(58.5)$

Repigmentation

Mixed

55 (67.9)

Present

Absent

4 (4.9)

Exacerbated ${ }^{* *}$

5

${ }^{\star}$ Phototherapy was commonly accompanied with topical therapy; ${ }^{* *}$ Four exacerbated cases were composed of 3 confetti and 1 sharp types of lesional borders. 
(b)

\begin{tabular}{|c|c|c|c|c|c|c|c|}
\hline $\begin{array}{l}\text { Initial inside } \\
\text { feature (n) }\end{array}$ & Repigmentation & $\begin{array}{c}\text { No } \\
\text { repigmentation }\end{array}$ & Perifollicular & Marginal & Diffuse & Mixed & $\begin{array}{l}\text { Repigmentation } \\
\text { frequency (\%) }\end{array}$ \\
\hline Clear white (13) & 5 & 8 & 1 & 2 & 0 & 2 & $38.5^{*}$ \\
\hline Faint (22) & 19 & 3 & 0 & 5 & 5 & 9 & 86.4 \\
\hline Multi-dot (23) & 18 & 5 & 3 & 1 & 1 & 13 & 78.3 \\
\hline Perifollicular (14) & 11 & 3 & 1 & 1 & 1 & 8 & 78.6 \\
\hline
\end{tabular}

${ }^{*}$ Indicates significantly lower repigmentation frequency compared to others ( $\mathrm{p}<0.05$ analyzed by Chi-square test).

referred to lesions that were a combination of the previous ones, specifically a combined perifollicular and marginal pattern [2]. The typing of initial and repigmented lesions was confirmed after careful review and agreement by 5 dermatologists. Repigmentation frequency after treatment was compared among 4 interior types and the significant difference was analyzed by Chi-square test.

Treatments included topical therapy, a combination therapy of topical and phototherapy, and systemic administration of steroids, among others. Topical drugs included steroids, topical calcineurin inhibitor, and vitamin D3, as shown in Table 1. All patients provided informed consent for this study, which was previously approved by the Osaka University Clinical Research Review Committee (accession \# 13421).

\section{Results}

Regarding gender, women accounted for $51.9 \%$ of cases and men accounted for $48.1 \%$ of cases. Their ages ranged from 1 to 78 years, with a median of 28.4 years. The most common clinical type was the segmental type, accounting for $44.4 \%$ of cases. The most frequently observed sites were the face and neck, at $58 \%$. Based on the Wood's light examination prior to treatment, the cases that supposedly had residual pigmentation, such as the faint, multi-dot, and perifollicular types, accounted for $68.8 \%$ of all cases. Overall repigmentation frequency after at least 3 months of treatment was $67.9 \%$. As shown in Table 1, out of the 4 cases with enlarged lesions, 3 were confetti-type, and one had a sharp-type border. Lesions with high activity, such as the confetti and trichrome types, accounted for $18.2 \%$ of total cases. Mixed-type repigmentation in combination with the marginal, diffuse, and perifollicular patterns, was the most common pattern after treatment among the cases that showed improvement (Table 1(a)).

We compared post-treatment repigmentation status based on the inner pattern prior to treatment. Table 1 (b) shows the 72 relevant cases that were followed up after treatment. Cases with "clear white" had the lowest repigmentation frequency (38.5\%), regardless of the lack of any significant treatment bias among lesions. Compared to cases with clear white, repigmentation frequency was significantly higher at $86.4 \%, 78.3 \%$, and $78.6 \%$ in cases with faint, multi-dot, and perifollicular, respectively although the border pattern prior to treatment was not significantly influenced in repigmentation frequency. The 
case with faint tended to present diffuse repigmentation pattern (26.3\%). Repigmentation frequency was lower at $55.6 \%$ in the group with leukotrichia compared to $73.8 \%$ in the group without leukotrichia.

\section{Discussion}

In this patient set, observation under a Wood's lamp revealed that $68.8 \%$ of cases exhibited incomplete white features inside their vitiligo lesions. We strongly suggest the potential utility of a Wood's light for identifying slight residual pigmentation that cannot be observed under white light, since there are no other similar reports of applying Wood's light to various types of vitiliginous patches for visual classification. A Wood's light can be clinically applied with minimal invasiveness, allowing for confirmation of the presence or absence of melanocytes at a wide angle and in detail [5] [6].

Repigmentation was observed in 53 out of 72 cases (73.6\%) that were followed up at least 3 months after treatment. Remarkably, while only $38.5 \%$ of the lesions classified as "clear white" type demonstrated any type of repigmentation after treatment, higher repigmentation frequency (81.4\%) was obtained among cases who presented residual pigmentation, thus indicating that the efficacy of treatment could be predicted by a Wood's light assessment prior to treatment. In addition, less repigmentation frequency was obtained in the group with leukotrichia compared that without leukotrichia, which is consistent with the opinion that leukotrichia is a poor prognostic indicator of repigmentaion (Data not shown) [7].

Observation with a Wood's light is reportedly useful for determining the therapeutic effect by identifying even slight repigmentation, which leads to an increase in the motivation of patients to continue treatment [8] [9]. However, there have been no investigations that objectively classified vitiligo lesions prior to treatment, as in the present study. Therefore, suggesting that short-term physical examinations are useful in the treatment of vitiligo is of great clinical significance. The confetti type is known to be an active sign of vitiligo [10] and was found in 3 out of 4 cases with exacerbated lesions, which suggests that early detection of lesions using a Wood's lamp can be considered for the early introduction of immunosuppressive agents, such as systemic immunosuppressants with half-pulse or oral steroids [11].

Several limitations associated with the present study warrant mention. First, our primary enrollment target was segmental before nonsegmental vitiligo in this study, because there were relatively few subjects, and their age corresponded predominantly to adolescence. Since those sites were considered suitable for assessments due to their cosmetic associations and of highest demand for treatment, we mainly evaluated face and neck lesions. This arbitrary site selection might have led to high repigmentation frequency and dynamic change in lesional features after treatment. Furthermore, several clinical confounding factors that influenced repigmentation were not excluded from this study, which may have 
interfered with the statistical analysis of the predictive values of Wood's lamp. Further assessment for increased number of vitiliginous lesions on equivalent treatment, site, and vitiligo type is required to clarify the above concerns.

\section{Conclusion}

We demonstrated that detailed observation under a Wood's lamp is useful for clearly classifying vitiligo lesions in this preliminary study, as well as the possibility for predicting not only disease prognosis but also repigmentation outcome at the vitiligo patients' first visit. A Wood's lamp observation is non-invasive and quite feasible in the daily clinical diagnosis of vitiligo patients. However, further studies are required to verify the results obtained in this preliminary classification of vitiligo lesions.

\section{Conflicts of Interest}

The authors declare no conflicts of interest regarding the publication of this paper.

\section{References}

[1] Ezzedine, K., Lim, H.W., Suzuki, T., Katayama, I., Hamzavi, C., Lan, C.C.E., et al. (2012) Revised Classification/Nomenclature of Vitiligo and Related Issues: The Vitiligo Global Issues Consensus Conference. Pigment Cell \& Melanoma Research, 25 E1-E13. https://doi.org/10.1111/j.1755-148X.2012.00997.x

[2] Ardigo, M., Muzio, F., Picardo, M. and Brazzelli, V. (2010) Vitiligo. In: Picardo, M. and Taieb, A., Eds., Non-Invasive Methods for Vitiligo Evaluation, Heidelberg: Springer Verlag, 183-195.

[3] Parsad, D., Pandhi, R., Dorga, S. and Kumar, B. (2004) Clinical Study of Repigmentation Patterns with Different Treatment Modality and their Correlation with Speed and Stability of Repigmentation in 352 Vitiliginous Patches. Journal of the American Academy of Dermatology, 50, 63-67. https://doi.org/10.1016/S0190-9622(03)00786-2

[4] Wang, Y.J., Chang, C.C. and Cheng, K.L. (2017) Wood's Lamp for Vitiligo Disease Stability and Early Recognition of Initiative Pigmentation after Epidermal Grafting. International Wound Journal, 14, 1391-1394. https://doi.org/10.1111/iwj.12800

[5] Bae, J.M. and Lee, R.W. (2019) 365-nm Narrowband Wood's Lamp for Vitiligo and Hypopigmentation Disorders. Journal of the American Academy of Dermatology. (Epub Ahead of Print). https://doi.org/10.1016/j.jaad.2019.08.064

[6] Takafuji, M., Tanemura, A., Hanaoka, Y., Arase, N., Kiyohara, E., Katayama, I., et al. (2019) Non-Invasive Evaluation and Differential Diagnosis for Pediatric Leukoderma in a Single Institute. JCDSA, 9, 313-320. https://doi.org/10.4236/jcdsa.2019.94028

[7] Benzekri, L., Ezzedine, K. and Gauthier, Y. (2013) Vitiligo Potential Repigmentation Index: A Simple Clinical Score that Might Predict the Ability of Vitiligo Lesions to Repigment under Therapy. British Journal of Dermatology, 168, 1143-1146. https://doi.org/10.1111/bjd.12147

[8] Alghamdi, K.M., Kumar, A., Taieb, A. and Ezzedine, K. (2012) Assessment Methods for the Evaluation of Vitiligo. JEADV, 26, 1463-1471. 
https://doi.org/10.1111/j.1468-3083.2012.04505.x

[9] Gawkrodger, D.J., Ormerod, A.D., Shaw, L., Mauri-Sole, I., Whitton, M.E., Watts, M.J., et al. (2008) Guideline for the Diagnosis and Management of Vitiligo. British Journal of Dermatology, 159, 1051-1076.

https://doi.org/10.1111/j.1365-2133.2008.08881.x

[10] Aboul-Fettouh, N., Hinojosa, J., Tovar-Garza, A. and Pandya, A.G. (2017) The Majority of Patients Presenting with Vitiligo Have a Clinical Sign of Activity. Journal of the American Academy of Dermatology, 77, 774-775.

https://doi.org/10.1016/j.jaad.2017.05.027

[11] Tovar-Garza, A., Hinojosa, J.A., Hynan, L.S. and Pandya, A.G. (2019) Addition of Oral Minipulse Dexamethasone to Narrowband Ultraviolet B Phototherapy and Topical Steroids Helps Arrest Disease Activity in Patients with Vitiligo. British Journal of Dermatology, 180, 193-194. https://doi.org/10.1111/bjd.17150

\section{Abbreviations}

TCI: topical calcineurin inhibitor 